\title{
Household Water Treatment Practice and Associated Factors Among People Living with HIV, Bahir Dar City Administration, Northwest Ethiopia
}

\author{
Amsalu Birara, ${ }^{1, *}$, Bikes Destaw ${ }^{2}$, Kefyalew Addis ${ }^{3}$ \\ ${ }^{1}$ Department of Environmental Health, School of Public Health Collage of Medicine and Health Sciences Bahir Dar University, Bahir Dar, \\ Ethiopia \\ ${ }^{2}$ Department of Environmental \& Occupational Health \& Safety, Institute of Public Health College of Medicine \& Health Sciences, \\ University of Gondar, Gondar, Ethiopia \\ ${ }^{3}$ Institute of Public Health College of Medicine \& Health Sciences University of Gondar, Gondar, Ethiopia
}

Email address:

amsalubirara@yahoo.com (A. Birara), ha.birara@gmail.com (A. Birara), bikesdestaw2004@gmail.com (B. Destaw),

kefadis@gmail.com (Kefyalew Addis)

${ }^{*}$ Corresponding author

\section{To cite this article:}

Amsalu Birara, Bikes Destaw, Kefyalew Addis. Household Water Treatment Practice and Associated Factors Among People Living with HIV, Bahir Dar City Administration, Northwest Ethiopia. International Journal of Biomedical Science and Engineering.

Vol. 6, No. 2, 2018, pp. 32-37. doi: 10.11648/j.ijbse.20180602.12

Received: May 23, 2018; Accepted: June 25, 2018; Published: July 16, 2018

\begin{abstract}
Introduction: PLHIV have substantially greater need for access to safe water, for bathing and washing soiled clothing and linen; safe drinking water is necessary for taking medicines. Therefore household water treatment is one of inexpensive and effective technology to make water safe. However the information of household water treatment practices and associated factors on these groups were inadequate. Objective: the: $t$ aim of the study was to assess household water treatment practice and associated factors among PLHIV who are member of the three associations in Bahir Dar city administration, Northwest Ethiopia. Methods: A Community based cross-sectional study was done among PLHIV from April-May 2013. A Simple random sampling technique was used to select the study participants. Pre-tested and structured questionnaire, observation checks list and residual chlorine test was used to collect the data. Binary and multivariate logistic regression analysis were used to determine the separate and confounding effect for variables with $p$-value $<0.2$ in binary analysis. Result: of the total respondents $76.3 \%$ reported that they treated their drinking water at home. But only $11 \%$ of respondents treated water at home within 24 hours of this study. The main reasons for not treating water at home were psycho-social problem 29 (53.7\%), unavailability of treatment methods $17(31.5 \%)$, and lack of knowledge how to use treatment methods $8(14.8 \%)$. Occupational status of the respondents $(\mathrm{AOR}=2.6 ; 95 \% \mathrm{CI} 0.162-0.903)$, duration of water storing time ( $\mathrm{AOR}=2.9 ; 95 \% \mathrm{CI}$ 1.471-5.692), and use of separate container for water storing ( $\mathrm{AOR}=3.1 ; 1.008-9.223$ ) were significantly associated variables with household water treatment practice. Conclusion: Household water treatment practices among people living with HIV were found low. Therefore the PLHIV needs special attention to improve household water treatment practice. fee free water treatment methods availability, promotion of household water treatment practice and participate in income generating activities are supreme important.
\end{abstract}

Keywords: Household Water Treatment, Water Treatment Practice, HIV, AIDS, Small Scale Water Treatment Methods

\section{Introduction}

Human Immune Virus/Acquired Immune Deficiency Syndrome/ HIV/AIDS /is one of the most devastating diseases in the world. By the end of 2011, the total number of world population which were affected by HIV/AIDS were estimated around 34 million and there were also 2.5 million new HIV infections. The burden of the epidemic continues to vary considerably between countries and regions. Sub- 
Saharan Africa remains the most severely affected region with nearly 1 person from every 20 adults, and $4.9 \%$ living with HIV which accounts $69 \%$ of the people living with HIV (PLHIV) worldwide [1].

Ethiopia is among the countries which is most affected by the HIV epidemic with an estimated adult prevalence of $1.5 \%$; it also has a large number of people living with HIV. Approximately 800,000 PLHIV and its prevalence is $1.9 \%$ women and $1 \%$ men respectively [2, 3]. HIV prevalence varies based on socio-economic characteristics, employment, residence, region, educational level, and wealth quintile of the people. Prevalence of HIV higher in women than men and somewhat higher among those who were employed than those who were not employed; in addition HIV prevalence is higher in urban areas accounting $4.2 \%$ than in rural areas accounting $0.6 \%$ [3].

Having safe drinking water and basic sanitation is the right of every human being. People need clean water and sanitation to keep up their health and dignity. Safe water and sanitation is essential in breaking the cycle of poverty since it improves peoples' health $[4,5]$. Even though PLHIV are particularly vulnerable to different disease due to inadequate water supply and poor sanitation therefore Diarrhea is a very common symptom of HIV/AIDS affected $90 \%$ of PLHIV and results in significant morbidity and mortality [6]. household water treatment and safe storage (HWTS) interventions improve water quality and reduce diarrheal disease incidence in developing countries [7]. All HIV-positive persons in the household encouraged treating their drinking water throughout their life, conversely, the information on water treatment and associated factors for these groups were limited. Hence, this study aimed to show the practices of HWT and associated factors among PLHIV. Thus, the findings of this study would serve to show the problem after implemented freely distributed treatment methods thereby very critical measures would be taken and by policy makers, and to know the progress of use of household water treatment so that it would aid to improve the health status of PLHIV.

\section{Methods}

A community based cross-sectional study were conducted by using quantitative method to assess household water treatment practices and associated factors among PLHIV, who were members of the three associations with a total of 2227 PLHIV (Tesfagohe=932, Mekidem Ethiopia $=1201$ and Wogagen=94) in Bahir Dar city administration from April-May, 2013. which is capital city of Amhara National Regional State and $565 \mathrm{Km}$ from Addis Ababa. It was located at 11o 38' latitude and at 37 o10' East longitudes at $1801 \mathrm{~m}$ above sea level. Bahir Dar is one of the tourist destinations in the country and strongly affected by HIV/AIDS epidemics.

\subsection{Sampling Procedures}

The study included all PLHIV who were members of the three PLHIV associations, and age greater than 18 years old. Simple random sampling technique was used to select the participants and random numbers were generated to increase randomness of the choice. From a total of 2227 PLHIV 459 study participants were selected. Participant who were scored the answered greater than the mean value of knowledge question are knowledgeable. Household water treatment practice were considered PLHIV who practiced at least one type of household (home based) water treatment methods within 24 hours and store the treated water in a narrow mouthed and covered container during the time of survey.

\subsection{Data Collection and Analysis}

The pretest and structured questionnaires used to collect the data. The questionnaires and checklists were first prepared in English and the English version of questionnaire was translated to local language [Amharic] and translated back to English by persons who are language professionals to keep up its consistency for real data collection. The questionnaire comprised socio demography, knowledge and water handling practice factor data's. Observation checks list and residual chlorine test were used to confirm the household water treatment practice. Data were checked, coded, and entered to Statistical Package for the Social Sciences (SPSS) version 20 and analysis were made by using Bivarate and multivariate logistic regression. Bivarate analysis using for each variable to know their significance with outcome variable and multivariate analysis were done for those variables which have cut point $\mathrm{p}$-value $<0.2$ in the Bivarate analysis to control confounding effect and determination of relationship between associated factors and household water treatment practice. Results were displayed using frequency tables in numbers, percentage, pie chart and bar graph. Odds ratio (OR) with $95 \%$ confidence interval was used to measure the strength of association and statistical significance was declared at $p$-value $<0.05$ to find the effect of factors on the outcome variable of household water treatment practice.

\subsection{Ethical Consideration}

Ethical clearance were obtained from the Institutional review board of the institution of public health, College of medicine and Health sciences, University of Gondar; Permission were obtained from Amhara regional health Bureau, Bahir Dar city administrative health office and Bahir Dar city HAPCO; Formal letters was prepared and dispatched to each respective PLHIV association. The entire study subject were informed about the objective, benefits and harms of participation in the study, and obtains their verbal consent before conducting data collection. They informed also have full rights of participating or not participating in the study; Confidentiality and privacy were ensured from all data collectors and principal investigator's side via using code numbers than names and keeping questionnaires locked. Data collectors interview separately from other people to keep the privacy of the clients. Data collectors gave health education about the benefit of home based water treatment practice and show how to treat and store their drinking water to the participant those who were done poor household water 
treatment practice during data collection.

\section{Results}

Socio-demographic characteristics of respondents

Out of the total 459 sampled households, 418 Household interviewed and Included in the analysis, which made the response rate $91 \%$. The mean age of respondents was 36.93 ( \pm SD 9.12) years. In terms of marital status $46.7 \%$ (195) were married.

The income distribution reveals that the poorest (lowest) $<200 \mathrm{ETB}$, second 200-300 ETB, third (middle) 300-400 ETB, fourth (high) 400-600 ETB and richest (highest) $>600$ quintile accounted for140 (35.5\%), 77 (18.4\%), 60 (14.4\%), 87 (20.8\%) and $54(12.9 \%)$, respectively, where Household families size 384 (91.9\%) was less than five person per house hold.

About the respondents unable to read and write were 173 (41.4\%), about the occupational status of the participants, house wife constitute117 (28\%). Majority of respondents were orthodox Christian 400 (95.7\%) and female 357 (85.4\%) (Table 1)

Table 1. Socio-demographic characteristic of PLHIV respondent in Bahir Dar city administration, April 2013 (n=418).

\begin{tabular}{|c|c|c|}
\hline Category & Frequency & Percentage \\
\hline \multicolumn{3}{|l|}{ Age } \\
\hline $18-24$ & 13 & 3.1 \\
\hline $25-44$ & 319 & 76.3 \\
\hline $45-64$ & 79 & 18.9 \\
\hline$\geq 65$ & 7 & 1.7 \\
\hline \multicolumn{3}{|l|}{ Sex } \\
\hline Male & 61 & 14.6 \\
\hline Female & 357 & 85.4 \\
\hline \multicolumn{3}{|l|}{ Marital status } \\
\hline Married & 195 & 46.7 \\
\hline Divorced & 110 & 26.3 \\
\hline Windowed & 86 & 20.6 \\
\hline Single & 24 & 5.7 \\
\hline Separated & 3 & 0.7 \\
\hline \multicolumn{3}{|l|}{ Educational status } \\
\hline Cannot read and write & 173 & 41.4 \\
\hline Can read and write only & 53 & 12.7 \\
\hline Have formal education & 192 & 45.9 \\
\hline \multicolumn{3}{|l|}{ Occupational status } \\
\hline House wife & 117 & 28.0 \\
\hline Merchant & 105 & 25.1 \\
\hline Daily worker & 84 & 20.1 \\
\hline Unemployed & 55 & 13.2 \\
\hline Employed & 54 & 12.9 \\
\hline Student & 3 & 0.7 \\
\hline \multicolumn{3}{|l|}{ Religion } \\
\hline Orthodox & 400 & 95.7 \\
\hline Muslim & 15 & 3.6 \\
\hline Protestant & 3 & 0.7 \\
\hline \multicolumn{3}{|l|}{ House hold member size } \\
\hline$\leq 5$ & 384 & 91.9 \\
\hline$>5$ & 34 & 8.1 \\
\hline \multicolumn{3}{|l|}{ House hold monthly income } \\
\hline Lowest (poorest) $<200 \mathrm{ETB}$ & 140 & 33.5 \\
\hline Second (poor) 200-300ETB & 77 & 18.4 \\
\hline Third (middle) $300-400$ ETB & 60 & 14.4 \\
\hline Fourth (High) 400-600ЕTB & 87 & 20.8 \\
\hline Fifth $($ Highest $)>600 \mathrm{ETB}$ & 54 & 12.9 \\
\hline
\end{tabular}

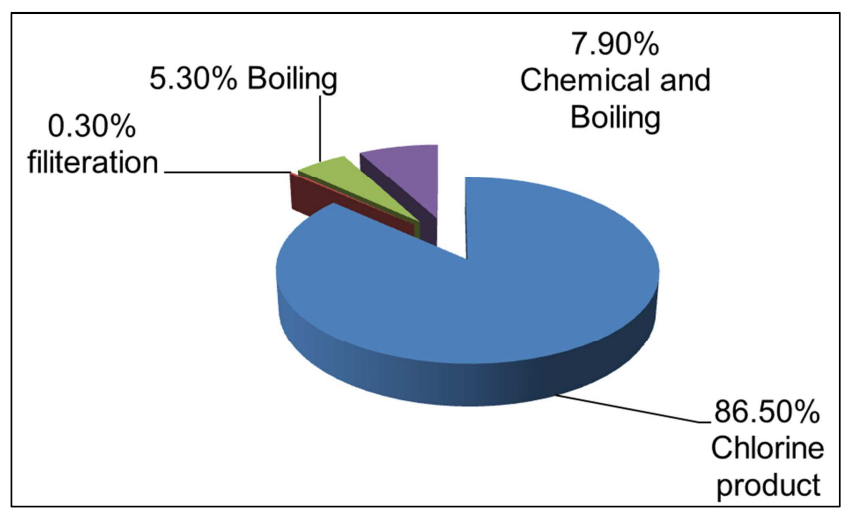

Figure 1. Methods of water treatment used by PLHIV in Bahir Dar city administration, April, 2013.

Table 2. Knowledge and water handling practice of the PLHIV in Bahir Dar city administration, May 2013. $(n=418)$.

\begin{tabular}{lll}
\hline Characteristics & Frequency & Percentage \\
\hline Knowledge about HHWT & & \\
Knowledgeable & 352 & 84.2 \\
Not knowledgeable & 66 & 15.8 \\
Store drinking water & & \\
yes & 412 & 98.6 \\
No & 6 & 1.4 \\
Duration of storage water & & \\
$>$ 2days & 117 & 28 \\
$\leq 2$ days & 301 & 72 \\
separate container for storage of water & & \\
Yes & 324 & 77.5 \\
No & 94 & 22.5 \\
Method of keeping drinking material & & \\
Properly & 315 & 75.4 \\
Improperly & 103 & 24.6 \\
Consumption of water per HH & & \\
$\leq 47$ liters per HH/ day & 263 & 62.9 \\
$>47$ liters per HH/day & 155 & 37.1 \\
Visiting of the association & & \\
Yes & 64 & 15.3 \\
No & 354 & 84.7 \\
Get education about HHWT from & & \\
association & & \\
Yes & 288 & 68.9 \\
No & 130 & 31.1 \\
Disclosure status & & \\
Yes & 408 & 97.6 \\
No & 10 & 2.4 \\
\hline
\end{tabular}

Multivariate analysis of associated Factors HHWT practice

All Variables which have cut point p-value $<0.3$ with household water treatment practice in Bivarate analysis selected and entered multivariate logistic regression analysis to identify the most important predictors of household water treatment practice.

About Respondents occupational status, those who are daily worker 2.6 times more likely their drinking water ( $\mathrm{AOR}=2.6 ; 95 \% \mathrm{CI} 0.162-0.903$ ) compared to those who are merchant; as well as Respondent who were storing their drinking water greater than two days were 3.1 times more likely to treat their drinking water at home $(\mathrm{AOR}=3.1 ; 95 \%$ CI 1.591-6.022) compared to those storing their drinking water less than or equal two days, and those respondents 
were use separate container for storing their drinking water 3.37 times more likely treat their drinking water at home;
(AOR=3.05; 95\% CI 0.228-1.939) compare to those use separate container for storing their drinking water (Table 3).

Table 3. Factors associated with house hold water treatment practice in Bahir Dar city administration, May 2013.

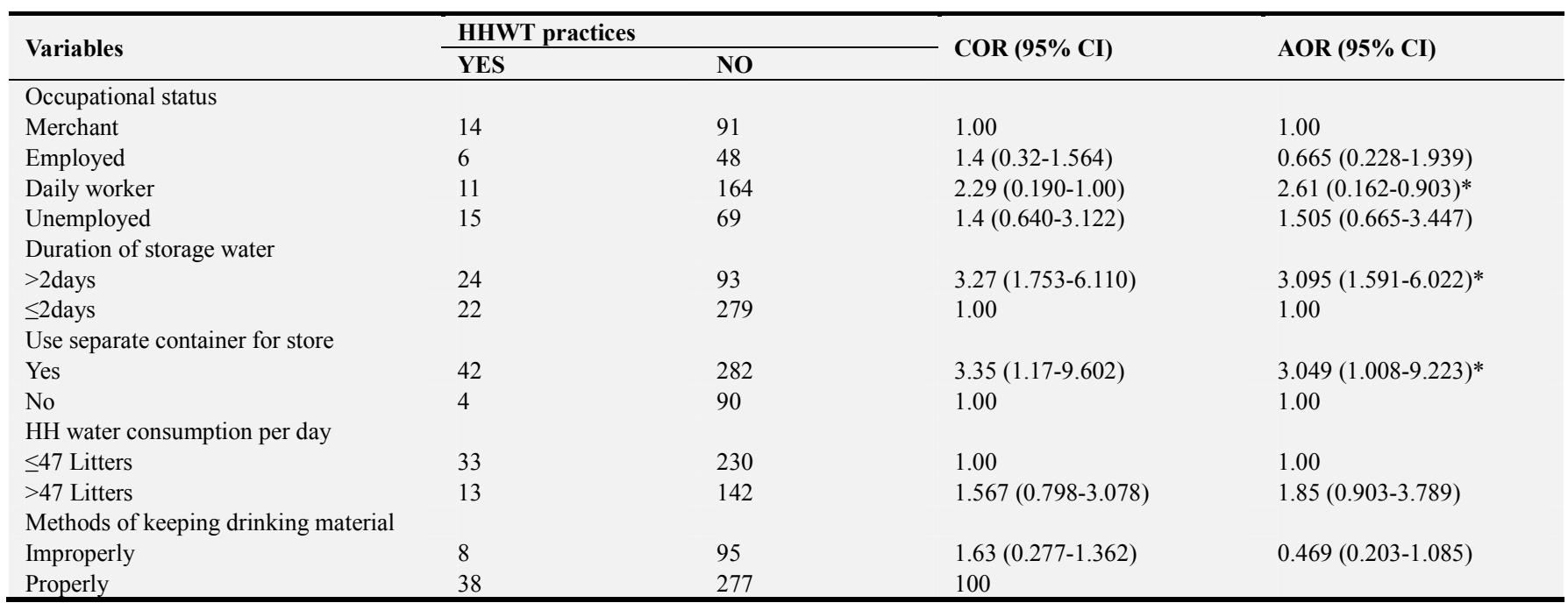

* Statistical significant at $\mathrm{p}<0.05$

\section{Discussion}

The need of safe water is important for many populations in developing countries. The most widely used (final) solution for the problem is to give centralized water treatment, but this approach is expensive, time-consuming, and will take decades to realize. To address immediate needs, other approaches are required while progress were made in improving household water treatment practice.

Household water treatment methods were used directly at homes of poor families and Immuno- compromised person so as to improve their drinking water quality; it is proven that an effective way to prevent water borne diseases. PLHIV needs special attention due to their vulnerability, to improve quality of life and to prevent diarrheal disease, Household water treatment and safe storage lets PLHIV acquire responsibility of their own water safety by treating and safely storing water themselves.

This study provides important information on household water treatment practice and associated factors with it. In this study the overall household water treatment practice was found $76.1 \%$. Out of these $11 \%$ treated their drinking water within 24 hours at the time of this study, samples taken from treated water, residual chlorine were only $8.7 \%$ compliance with standard, $54.3 \%$ above and $37 \%$ below from standard of WHO drinking water guideline. This also could be the evidence of that the shortage of the municipal water treatment system.

Among the potential determinants explored about to household water treatment practice of PLHIV who are a member of the three PLHIV associations in Bahir Dar city administration, occupational status, duration of water storing time, and use separate container for storing drinking water are significant predicators of household water treatment practice.
In this study household water treatment practice within 24 hour was found $11 \%$ which less than studies conducted in Nepal and Bolivia which was $57.1 \%$, 38\% respectively [11, 28]; and Malawi, Zambia and Pre-Urban Zambia which was, $43.1 \%, 33 \%$ and $47 \%$ treated their drinking water [15]. The possible reason for such difference could be source of water supply, health strategies, perception of people accepted household water treatment methods, safe water coverage and availability of country regulation, policies and targets of scaling up of household water treatment practice.

The result was similar in studies conducted in Addis Ababa and in Gondar city administration 11\% and $10.9 \%$ respectively $[19,20]$. I think Ethiopia have policies, targets, committee, regulation and certification to scaling up of household water treatment and safe storage practice in nationwide [29]. Even though FMOH, HAPCO PLHIV association done greater effort providing treatment method especially Wuha agar and 20 liter Jerricans together with others materials freely for all PLHIV starting from 2009, there was no improvement in the practice of household water treatment of PLHIV.

When we come to treatment method of this study, $86.5 \%$ were using chlorine product especially Wuha Agar, which is most widely used for most households those practiced household water treatment methods. Boiling 5.3\%, Filters 1 $0.3 \%$ and both chlorine and boiling alternatively $7.9 \%$.

When we check this study with Bolivia, Utilization of chlorine product of this study is greater, but utilization of boiling, filtration and both chemical and boiling alternatively less in Bolivia; which is account $31 \%, 33 \%, 23 \%$, and $8.3 \%$, respectively [28]. Such types of discrepancy possibly, boiling is time consuming, exhausting task, and unavailability of fire wood, lack of money to buy filtration materials, source of water supply, safe water coverage and means of getting treatment methods. 
This study is comparable with study in Uganda $88 \%$ of them used water Guard (Wuha Agar), $8.3 \%$ of them use both Boiling and chemical product [13].

In this study utilization of Wuha Agar is greater than study conducted in Addis Ababa and Gondar city administration were accounts $62.5 \%, 6.2 \%$, respectively $[19,20]$. These show that use of Wuha agar is better than Addis Ababa and Gondar. Even though Wuha agar distributed freely, and FMOH, HAPCO, PLHA association and world vision (supplier) had great effort, utilization of household water treatment methods there was no improvement.

At household level a lot of effort were done to make the water safe, like collect, transport and treated their drinking water. If, treated water is not handled and stored properly, quality of treated water would possibly become more affected (poorer) than the source water and might cause people to get sick.

Many countries of Africa used 20 liter Jerricans which are initially used to transport vegetable cooking oils, and they would be cleaned and used to transport and store water [7].

about this study $98.6 \%$ of household stored their drinking water, out of which $89.7 \%$ put in plastic Jerricans and from these $53.6 \%$ have spigot (faucet) and $46.4 \%$ have not; $10 \%$ in traditional clay pots (Insera), and remaining $0.2 \%$ stored in metal containers;

This is alike to a study carried out in Zambia $98 \%$ of the respondents use Jerricans for storing their water while the remaining $2 \%$ use metal containers [15]. While a study which was conducted in Malawi shows that $48.3 \%$ of the respondents store their water in clay Jars, $41.7 \%$ of the respondents reported that they use plastic containers, and the remaining $8.3 \%$ of the respondents use metal containers [17].

This variation might be safe storage promotion strategies of the country, climatically difference of the country, perception of people who clay Jar has potential to cool drinking water and awareness of people about the effect of unsafe storage of drinking water.

This is similar study conducted in Gondar $96.94 \%$ of the households store their drinking water out of which $90.2 \%$ used plastic Jerricans, $10.8 \%$ of them stored in traditional clay pots (Insera) and the remaining $1.7 \%$ stored in metal containers [20]. Considering that they got freely from health institution, supplied by world vision, use of Jerricans of PLHIV for water storage was low.

Among the potential determinants explored about to household water treatment practice, from sociodemographics characteristics of respondents, occupational status were significant predictors of household water treatment practice. This possibly people's those who are daily worker with living HIV fear of diarrhea disease and other infectious disease than those who are merchant living HIV.

Duration of water storing time significant factors of household water treatment practice, this is possibly respondent perception which means water store more than two days become polluted other than they fetch often and they perceive that water is safe and one of the reason did not treat their drinking water were perception of people who municipal water was safe. These are also probably avoiding shortage of water to use other purpose, and water is available normally, this encourages that treating water and utilizing treated water.

Use of separate container for storing drinking water are also other significant predictors of house hold water treatment practice this might be people have better methods of storing drinking water perceived that drinking water may not contaminate during the container use for other domestic purpose, and safe storage is one of drinking water treatment methods as well as means of avoids cross contamination.

Strengths of the study

The study focused on the most vulnerable segment of the population. Residual chlorine test were done could be sure whether treatment were carried or not in addition to using observation methods for water treated by using chlorine product.

Limitation

The cross sectional nature of this study may not show a real causal association and the study not includes all PLHIV live in Bahir Dar city administration.

\section{Conclusion}

Household water treatment practices among people living with HIV in the member of the association were found low. Among the potential determinants explored regarding practice of household water treatment, PLHIV who are a member of three PLHIV associations in Bahir Dar city administration, occupational status of respondents, duration of water storage time, and means of keeping drinking materials were found independent factors of household water treatment practice.

\section{Recommendation}

Amhara Regional Health Bureau and HIV/AIDS Prevention and Control office and other partner work closely to increase the practice of household water treatment methods on PLHIV by enhancing training for volunteers, and Staff of PLHIV associations towards water safety, use of household water treatment methods. They have to develop control mechanism to efficient use of resource especially freely distributed water treatment methods. PLHIV, better to use freely distributed household water treatment methods properly like "Wuha Agar" and a narrow necked water container with spigot.

\section{Author's Contribution}

Amsalu Birara conceptualized the research problem, designed the study, conducted field work, collected data, data analysis and drafted the manuscript.

Bikes Destaw was involved in preparing the research proposal, data analysis and research report, and revision of the manuscript.

Kefyalew Addis was involved in revision of the research 
proposal, data analysis and revision of the manuscript for publication. All authors of the manuscript have read and agreed to its content.

\section{Acknowledgements}

We would like to thank University of Gondar and Ethiopian food medicine and Health care administration and control authority for providing this opportunity.

We would like to thank Ethiopian Food, Medicine and Health care administration and control authority.

I would like to thank Mr. Derje Birhanu for devoting his time and valuable comment

We would like to appreciate PLHA associations in Bahir Dar city administration, data

Collectors and supervisors and the participants.

\section{References}

[1] UNAIDS. UNAIDS Report on the global AIDS epidemic |. 2012.

[2] HPACO F. Country Progress Report on HIV/AIDS Response, 2012.

[3] CSA. Ethiopia Demographic and Health Survey. 2011.

[4] WHO. UN-Water Global Analysis and Assessment of Sanitation and Drinking-Water. The challenge of extending and susta ining servicesGLAAS REPORT. 2012.

[5] CAWST. INTRODUCTION TO DRINKING WATER QUALITY TESTING A CAWST TRAINING MANUAL. 2009.

[6] Katabira ET. Epidemiology and management of diarrheal disease in HIV-infected person. int J Infected dis. 1999 (3:):164-7.

[7] CDC. SAFE WATER FOR THE COMMUNITY, A Guide for Establishing a Community-Based Safe Water System Program. 2008.

[8] JRL, et. al. EFFECT OF HOME-BASED WATER CHLORINATION AND SAFE STORAGE ON DIARRHEA AMONG PERSONS WITH HUMAN IMMUNODEFICIENCY VIRUS IN UGANDA. Am J Trop Med Hyg. 2005; 73 (5):926-33.

[9] Kmminga. E. HIV/AIDS and water, sanitation and hygiene, Thematic Overview Paper. 2005.

[10] WHO U. Drinking Water Equity, safety and sustainability. 2011.

[11] Wateraid. Access to water, sanitation and hygiene for people living with HIV and AIDS: A cross-sectional study in Nepal. 2010 .

[12] Anderson B, Romani J, Wentzel M, Phillips H. Awareness of Water Pollution as a Problem and the Decision to Treat Drinking WaterAmong Rural African Households with Unclean Drinking Water: South Africa.
[13] COLINDRES R, MERMIN J, EZATI E, KAMBABAZI S, BUYUNGO P, SEKABEMBE L, et al. Utilization of a basic care and prevention package by HIV-infected persons in Uganda. AIDS Care. 2008; 20 (2):139-45.

[14] Peletz. R, et. al. Drinking Water Quality, Feeding Practices, and Diarrhea among Children under 2 Years of HIV-Positive Mothers in Peri-Urban Zambia. 2011.

[15] Kangamba M, Roberts C, Campbell J, Service J, Adalla C. WATER \& SANITATION ASSESSMENT OF HOMEBASED CARE CLIENTS IN ZAMBIA. 2006.

[16] CR. Services Integration of water, sanitation and hygiene into HIV programs: Lessons from Malawi. 2009.

[17] LOCKWOOD K, MSAPATO K, SENEFELD S, NOGI J, PERRIN P, MTIKA M. WATER \& SANITATIONASSESSMENT OF HOMEBASED CARE CLIENTS IN MALAWI. 2006.

[18] Ezra J. Barzilaya TSA, Elizabeth M. Blantona, Abiodun A. Akinpelumic,, Matthew E. Coldirond OA, Olufolake A. Adeleyeb, Amy LaTriellec, Robert M. Hoekstrae,, Quick UGaR. Diarrhea prevention in people living with HIV: an evaluation of a point-of-use water quality intervention in Lagos, Nigeria. AIDS Care. 2011; 23 (3):330-9.

[19] SIYOUM M. Assement on water, sanitation and hygiene status among home based care clients of PLWHA in Addis Ababa. 2009.

[20] Yellow. w et. al. Assessment of water, sanitation, and hygiene practice and associated factors among people living with HIV/AIDS home based care services in Gondar city, Ethiopia. BMC Public Health. 2012; 12:1057.

[21] Organ J. WATER AND HIV: WORKING FOR POSITIVE SOLUTIONS, Impacts of the HIV epidemic on Access to Safe Water, Sanitation and Hygiene in the Copperbelt of Zambia.

[22] WSP. Water, Sanitation, and Hygiene for People Living with HIV and AIDS, Field Note 2007.

[23] USAID. Global Development Alliance, Safe Drinking Water Alliance, Experiences in Haiti, Ethiopia, and Pakistan:Lessons for future water treatment programs. 2008.

[24] Services CR. Water, Sanitation and Hygiene Considerations in Home-Based Care for People Living with HIV. 2010.

[25] Mugambe R, Tumwesigye N, Larkan F. Barriers to accessing water, sanitation and hygiene among people living with HIV/AIDS in Gomba and Mpigi districts in Uganda. 2012.

[26] Bahir Dar City Adminstration water office. Yearly report 2012.

[27] Bureau of Finance and Economic developement. 2012/2013. Plan.

[28] QUICK R, VENCZEL L, MINTZ E, SOLETO L, APARICIO J, GIRONAZ $M$, et al. Diarrhoea prevention in Bolivia through point-of-use water treatment and safe storage: a promising new strategy. Epidemiol Infect. 1999; 122:83-90.

[29] WHO. Status of National Household Water Treatment and Safe Storage Policies in Selected Countries Results of global survey and policy readiness for scaling up. 2012. 